\title{
Aplicación de andamiaje en un modelo de aprendizaje sostenible y su influencia en el estado emocional durante la COVID-19 Scaffolding in a sustainable learning environment and influence on emotions during COVID-19
}

\author{
Ricardo de Arriba Laso ${ }^{1}$, Maximiliano Paredes-Velasco ${ }^{2}$ \\ r.dearriba.2020@alumnos.urjc.es,maximiliano.paredes@urjc.es \\ ${ }^{1}$ Máster en Formación del Profesorado, \\ Informática y Tecnología \\ Universidad Rey Juan Carlos \\ Madrid, Spain \\ 2 Departamento de Ciencias de la Computación, \\ Arquitectura de la Computación, Lenguajes y \\ Sistemas Informáticos y Estadística e \\ Investigación Operativa \\ Universidad Rey Juan Carlos \\ Móstoles, Madrid, Spain
}

Resumen- Las emociones son un factor determinante en el aprendizaje, especialmente en la actual situación de pandemia. En este entorno, los estudiantes han estado sometidos a una elevada presión, pudiendo afectar negativamente a sus emociones. Actitudes como la desvinculación con la tarea y escasa autonomía se han visto agravados. El andamiaje (scaffolding) tiene el propósito de ayudar a los alumnos a realizar tareas que no serían capaces de hacer por sí mismos e ir transfiriendo la responsabilidad de resolución de forma gradual, lo cual puede repercutir positivamente en las emociones. En este artículo se propone un modelo de aprendizaje sostenible con integración de andamiaje y apoyado por el uso de plataformas abiertas. Se ha evaluado la efectividad y facilidad de uso de este modelo con un grupo de alumnos, así como su influencia en las emociones. Como resultados principales podemos afirmar que el modelo de aprendizaje con andamiaje, en el contexto implantado, ha mejorado la adquisición de conocimiento en diferentes niveles de la taxonomía de Bloom y el estado emocional del estudiante, $\mathrm{y}$ ha reducido la percepción de dificultad de las tareas. Además, se ha observado que las percepciones (de dificultad, así como de utilidad del andamiaje) están relacionadas con las emociones que experimentan los estudiantes.

Palabras clave: Andamiaje, Aprendizaje sostenible, Emociones, STEM

\begin{abstract}
Emotions are a determining factor in learning, especially in the current pandemic situation. In this environment, students have been under high pressure, which can negatively affect their emotions during learning. Attitudes such as disconnection from the task and little autonomy have been aggravated. The idea of scaffolding is intended to help students carry out tasks that they would not be able to do on their own and gradually transfer responsibility for the resolution, which can have a positive impact on emotions. This article proposes a sustainable learning model with scaffolding integration and supported by the use of open platforms. The effectiveness and usability of this model have been evaluated with a group of students, as well as its influence on emotions. As main results, we can affirm that the scaffolding learning model, in this context, improves the acquisition of knowledge at different levels of Bloom's taxonomy, improves the emotional state of the student and reduces the perception of task difficulty. In addition, it has been observed that the perceptions (about task difficulty and scaffolding usefulness) are related to the emotions experienced by the students.
\end{abstract}

Keywords: Scaffolding, Sustainable learning, Emotions, STEM

\section{INTRODUCCIÓN}

Los objetivos fijados por la Unión Europea para el año 2020 recogen la necesidad de incrementar el nivel de formación y cualificación tanto de los jóvenes en edad escolar como de la población trabajadora, para lo que es necesario reforzar, modernizar y flexibilizar las enseñanzas de formación profesional (Real Decreto, 2011). En esta última década, nuestro país ha sufrido una importante crisis económica, con el consecuente aumento del paro, agravado por la crisis sanitaria actual debido a la pandemia de la COVID-19. Todo ello hace que la reinserción laboral de jóvenes y profesionales sea un objetivo prioritario para nuestra sociedad. La formación profesional juega un papel fundamental para reajustarnos en esta nueva situación que vivimos y debe preparar al alumnado ante este nuevo panorama social y laboral.

Uno de los grandes pilares que se debe potenciar en la formación es la capacidad de los alumnos de aprender a aprender (Delors, 1994). Para ello se debe dotar a los alumnos de autonomía y facilitarles un traspaso de conocimientos gradual que no conduzca a sentimientos de rechazo o ansiedad. Las emociones del estudiante son un factor determinante en el aprendizaje, teniendo un papel especial en la actual situación de pandemia. Mejorar el rendimiento académico e incrementar la motivación de los estudiantes de Formación Profesional son retos que la metodología docente tradicional no suele conseguir debido a la presentación de contenidos monolíticos y con escasa ayuda tecnológica. La intervención del docente en el proceso de aprendizaje es poco efectiva y demasiado centrada en la enseñanza (el docente) en lugar del aprendizaje (los alumnos).

El apoyo que el docente puede aportar al alumno durante este proceso puede suministrarse en la forma de andamiaje (scaffolding). El andamiaje se basa en la transferencia de conocimiento y estrategias de ejecución entre docente y alumno, a medida que este último va adquiriendo mayor grado de autonomía (Wood et al., 1976). La responsabilidad de la tarea a ejecutar va siendo asumida por el aprendiz con la ayuda 
de su maestro, la cual se va retirando poco a poco hasta que el alumno pasa a ser totalmente independiente. El andamiaje se diseña normalmente para potenciar los niveles de aprendizaje más elevados (high-order thinking skills) (Kim et al., 2018), que de acuerdo con la taxonomía de Bloom (1956) revisada por Krathwohl, son los niveles de análisis, evaluación y síntesis (Krathwohl, 2002).

El docente tiene el reto de ser un guía eficaz en este proceso de transformación del alumno en el andamiaje y su eficacia, puede incrementarse sensiblemente mediante el uso de las Tecnologías de Información y Comunicación (TIC), especialmente en un ambiente de transformación tecnológica acelerada como el que vivimos por la pandemia de la COVID19. Soluciones de aprendizaje semipresencial y basadas en el uso de plataformas de software libre pueden ser grandes aliados tanto de docentes como de estudiantes.

El objetivo de este trabajo es proponer un modelo de aprendizaje de andamiaje eficaz y fácil de usar que mejore la adquisición de conocimiento y el estado emocional del estudiante. Este modelo es apoyado por el uso de plataformas abiertas, proporcionando un aprendizaje sostenible y accesible. Para validar este modelo, se ha realizado una experiencia con alumnos de formación profesional en un contexto COVID-19, donde se combinaban clases presenciales y online para respetar las restricciones sanitarias, evaluándose tanto la percepción de facilidad de uso (usability) y emociones de los estudiantes, como los resultados de aprendizaje. Aunque el modelo de aprendizaje se ha creado y aplicado en educación en formación profesional en el contexto COVID-19, el diseño de éste permite que se pueda aplicar a otros entornos educativos diferentes, adaptando el feedback del andamiaje en relación a los contenidos de formación.

El resto de la contribución describe en primer lugar los principales aspectos de andamiaje y la influencia de las emociones en el aprendizaje. A continuación, se describe el modelo de aprendizaje propuesto y la experiencia realizada junto con los resultados obtenidos. Finalmente, se exponen las conclusiones de este trabajo.

\section{SCAFFOLDING Y EMOCIONES EN EL APRENDIZAJE}

El andamiaje se enmarca en la perspectiva del aprendizaje socioconstructivista (Vigotsky, 1979). El socioconstructivismo considera que el aprendizaje se produce por la interacción social y se basa en el concepto de Zona de Desarrollo Próximo (ZDP), que refleja la distancia entre el nivel de desarrollo actual del aprendiz y su nivel de desarrollo potencial, definido por la resolución de tareas bajo la supervisión de una persona más capacitada. La idea de andamiaje se traslada al ámbito escolar con el propósito de ayudar a los estudiantes a llevar a cabo tareas que no serían capaces de realizar por sí mismos e ir transfiriendo la responsabilidad de la resolución de la tarea de forma gradual (Pérez \& Aleixandre, 2018). El apoyo docente debe cumplir tres características para ser considerado andamiaje (Van de Pol et al., 2010): contingencia, es decir, adaptación al nivel del alumnado; temporalidad, lo que implica que el apoyo se debe ir retirando de forma gradual a lo largo del tiempo; y transferencia de responsabilidad al alumnado de forma gradual. Existen cuatro categorías de andamiaje (Kim et al., 2018): conceptual, estratégico, metacognitivo y motivacional. Este puede suministrarse entre semejantes, por un experto o mediante apoyo tecnológico (Belland, 2017). Las estrategias que comúnmente se usan durante el andamiaje son: la retroalimentación, las pistas, las preguntas, las instrucciones, las explicaciones y la modelización.

Además, el estado emocional de los alumnos es un factor fundamental para el aprendizaje significativo (Lacave et al., 2020). Por un lado, la emoción predispone para el proceso de atención y por otro, produce una vinculación (o desvinculación) con el contenido. Las emociones que los alumnos desarrollan durante cualquier método de aprendizaje ofrecen una información muy valiosa para el ajuste del método docente. Para medir las emociones, existen varios cuestionarios o escalas como es la escala PANAS (Positive And Negative Affect Schedule), la cual clasifica las emociones en positivas y negativas, o la escala AEQ (Achievement Emotions Questionnaire), que mide las emociones más específicamente, constituyendo una escala validada en contextos académicos (Paolini et al., 2014). Esta escala mide emociones como la esperanza, el orgullo, el enfado, la ansiedad, la vergüenza, la desesperanza, el aburrimiento o el disfrute. Lo hace mediante ochenta preguntas relacionadas con estas emociones descritas y se puede usar para medir el estado emocional durante tres momentos: antes, durante y después del aprendizaje.

\section{PRopuesta de UN MOdELO SOSTENIBLE DE APRENDIZAJE}

El modelo de aprendizaje semipresencial que se ha diseñado se sustenta sobre dos pilares: primero, presentación de los contenidos de manera escalonada y fomento de la autonomía del estudiante (andamiaje y Taxonomía de Bloom); y segundo, uso de una plataforma digital libre que proporciona accesibilidad en cualquier lugar y en cualquier momento.

\section{A. Estructura del modelo con andamiaje}

El modelo de aprendizaje consta de tres fases secuenciales que son descritas a continuación.

Fase de Teoría. En esta primera fase se presenta a los alumnos de manera síncrona una introducción sobre los contenidos de forma teórico-práctica, mediante explicación y modelado del profesor.

Fase de Actividad. En esta fase los alumnos deben resolver tres retos o actividades que se deben entregar al profesor. Estas actividades están enmarcadas en los siguientes crecientes niveles de conocimiento: recordar, analizar y sintetizar, de acuerdo con la taxonomía de Bloom revisada (Krathwohl, 2002). Estas actividades se integran en la plataforma tecnológica de acceso libre basada en la web para que los alumnos puedan ir desarrollándolas a su ritmo y en cualquier lugar. Dentro de los niveles más complejos, análisis y síntesis, se integra un andamiaje tecnológico (Computer-based scaffolding), basado en la estrategia de pistas y preguntas y de tipo metacognitivo y estratégico. El andamiaje se va retirando (fadding) y es personalizable (self-selection) por cada alumno en función de sus necesidades y capacidades particulares. Durante la resolución de estas actividades los alumnos pueden hacer uso de herramientas de comunicación como el chat o foro virtual para comentar con sus compañeros o con el docente. Después de que los alumnos hayan entregado estas tres actividades, se les muestra una retroalimentación automática en función de sus respuestas. Esta retroalimentación ha sido previamente integrada por el profesor en la plataforma tecnológica. 
Fase de Retroalimentación. Esta es la tercera y última fase, en la que los alumnos exponen al profesor sus preguntas y dudas de forma síncrona, ayudándose de la retroalimentación que han recibido durante el final de la segunda etapa. El profesor responde a los alumnos estas preguntas y los alumnos terminan de trabajar las partes de las actividades de la segunda fase que no fueron capaces de resolver completamente si hubiese sido el caso. El profesor da retroalimentación a los alumnos en función de lo que estos requieren durante la clase, desarrollando así scaffolding profesor-alumnos basado en retroalimentación. Finalmente, el profesor y los alumnos realizan una retrospectiva sobre las actividades de la segunda fase.

\section{B. Plataforma tecnológica}

Teniendo en cuenta el modelo de aprendizaje descrito anteriormente, se ha desarrollado una herramienta basada en Google Forms e integrada en Google Classroom, proporcionando así una plataforma libre y accesible de apoyo al modelo de aprendizaje. Esta plataforma, basada en la web y de acceso gratuito, permite integrar funcionalidades de aula virtual con ayuda de sus aplicaciones. En este modelo propuesto, se usa fundamentalmente su chat, tablón de anuncios y foro para mantener viva y facilitar la comunicación entre docente y alumnos en un entorno tan difícil como el derivado de la COVID-19 (ver Figura 1). Por otro lado, mediante la herramienta desarrollada con Google Forms, se apoya el desarrollo de los retos y actividades, integrándose así el andamiaje tecnológico (ver Figura 2). Se ha guiado el flujo de navegación en función de las respuestas de cada alumno. De acuerdo con éstas, el andamiaje (basado en pistas y preguntas) se va retirando o se mantiene. $\mathrm{Si}$, por ejemplo, un alumno responde acertadamente al grupo de preguntas y pistas, se retira ese andamiaje y se procede a la resolución final. En caso contrario, se siguen mostrando más bloques de andamiaje. Al enviar las respuestas, el alumno recibe un feedback para cada una de sus contestaciones.

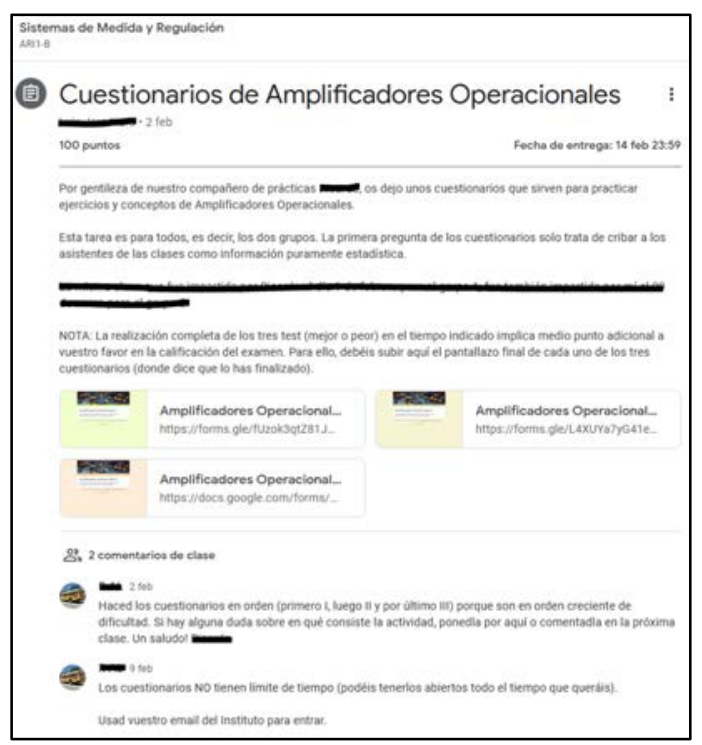

Figura 1 - Tablón de anuncios en la Fase de Actividad del modelo (vista del alumno)

De esta forma se proporciona un modelo de aprendizaje sostenible y accesible, sin necesidad de costosos desarrollos ni de inversiones tecnológicas específicas por parte de los centros educativos, utilizando plataformas abiertas con el único requisito de disponer de conexión a Internet. Su acceso por HTTP a través de un simple navegador Web le confiere un carácter de accesibilidad universal.

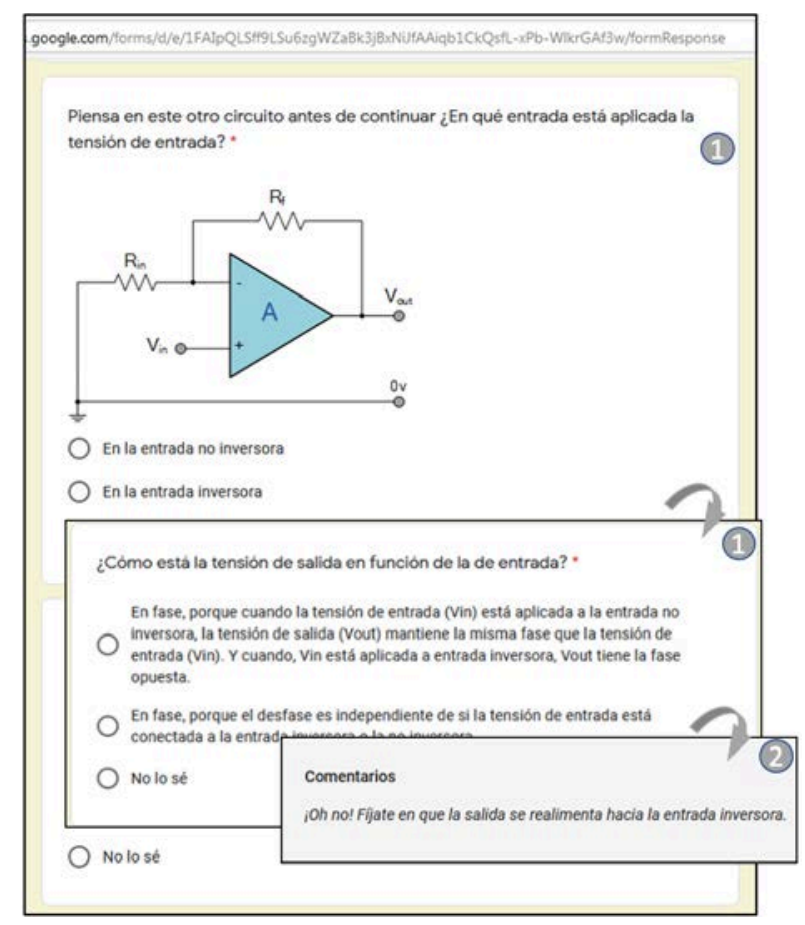

Figura 2 - Ejemplo de andamiaje secuencial integrado en Forms, basado en preguntas (marca 1) y feedback (marca 2) durante la Fase de Actividad del modelo

\section{EXPERIENCIA}

Con el objetivo de validar la facilidad de uso del modelo educativo y su eficacia en el aprendizaje, se realizó una experiencia con estudiantes en pleno contexto COVID-19 vivido durante el curso 2020-2021. En esta situación extrema, el estado emocional del estudiante es vital, por lo que se prestó especial atención al estudio de las emociones en el proceso de aprendizaje.

\section{A. Objetivo y contexto educativo}

Las preguntas de investigación junto con las respectivas hipótesis fueron las siguientes:

RQ1. ¿El modelo de aprendizaje propuesto facilita la adquisición de conocimiento?

H1. El uso de andamiaje en el modelo de aprendizaje propuesto mejora la adquisición de conocimiento en distintos niveles de la taxonomía de Bloom.

RQ2. ¿El modelo de aprendizaje propuesto es fácil de usar (usability) para los estudiantes?

H2. La percepción de dificultad sobre la tarea y la percepción de utilidad sobre el andamiaje y el modelo de aprendizaje empleado están relacionadas con las emociones de los alumnos.

H3. El andamiaje del modelo propuesto mejora el estado emocional de los alumnos y reduce su percepción de dificultad.

La experiencia se lleva a cabo en el IES Julio Verne de Leganés, dentro del ciclo formativo de Grado Superior en 
Automatización y Robótica Industrial. Intervinieron 36 alumnos organizados en dos grupos: un grupo experimental (GE) de 21 alumnos que utilizó el modelo de aprendizaje propuesto en el módulo de Sistemas de Potencia (aplicación de andamiaje), y un grupo de control (GC) de 15 alumnos que utilizó la metodología tradicional de clase magistral en el módulo de Robótica Industrial (sin andamiaje). La plataforma de Google Classroom estaba ya integrada en el centro formativo por lo que no fue necesario ningún coste para su implantación. Debido a los límites de aforo impuestos por la COVID-19, los alumnos asistieron presencialmente solamente la mitad de las horas lectivas que en un curso normal.

\section{B. Variables e instrumentos}

A continuación, se resumen los instrumentos y variables medidas durante la intervención. Los nombres de algunas variables pueden contener sufijos, los cuales tienen el siguiente significado: PRE, indica que es una variable de un pre-test; POS_INTER, es una variable de un test intermedio, y POS FINAL o POS, que indican que es una variable de un posttest. Las escalas utilizadas son las siguientes:

1) Escala de conocimiento. Los conocimientos desarrollados por los alumnos han sido medidos a través de la calificación de las preguntas planteadas en las actividades. Se ha utilizado preguntas tipo test y se ha usado rúbrica de corrección en los casos necesarios de preguntas con respuesta abierta, ponderando la nota de 1 a 10 . Las variables medidas con esta escala agrupadas por niveles son: ConocimientoRecordar_POS_INTER y POS_FINAL; ConocimientoAnalisis_PRE, POS_INTER y POS_FINAL; y ConocimientoSintesis_POS_INTER y POS FINAL.

2) Escala de percepción de dificultad. Las percepciones de los alumnos en cuanto a la dificultad de la actividad se han planteado con cuatro preguntas en escala Likert del 1 al 5. Las variables medidas han sido PercepDificultad_PRE y PercepDificultad_POS.

3) Escala de percepción de utilidad. Las percepciones de los alumnos en cuanto a la utilidad del andamiaje recibido se han planteado con dos preguntas en escala Likert del 1 al 5. Las variables medidas han sido PercepUtilidadScaff_TECNO y PercepUtilidadScaff_PROF.

4) Escala de emociones. Las emociones se han medido con quince ítems de una parte de la escala AEQ, en concreto los correspondientes a la evaluación de después del aprendizaje, usando una escala de Likert del 1 al 5. Las variables medidas son: Ansiedad, Vergüenza, Enfado, Orgullo, Desesperanza y Disfrute.

\section{Método}

La intervención llevada a cabo se realizó en tres etapas correspondientes a las tres fases del modelo de aprendizaje propuesto. Con el fin de poder dar respuesta a las hipótesis sobre la adquisición de conocimientos en distintos niveles de la Taxonomía de Bloom, se han dispuesto varios test intermedios para los niveles de recordar, análisis y síntesis, y además al final de toda la experiencia se realizó un post-test final para estos tres niveles. Así mismo, se evalúa al finalizar la experiencia el estado emocional de los alumnos (con un subconjunto del AEQ), sus percepciones sobre la utilidad del método docente y la dificultad. En la Figura 3 se muestra un resumen de las etapas, actividades y secuenciación temporal realizada, donde se puede ver la planificación tanto para el grupo GE como el grupo GC.

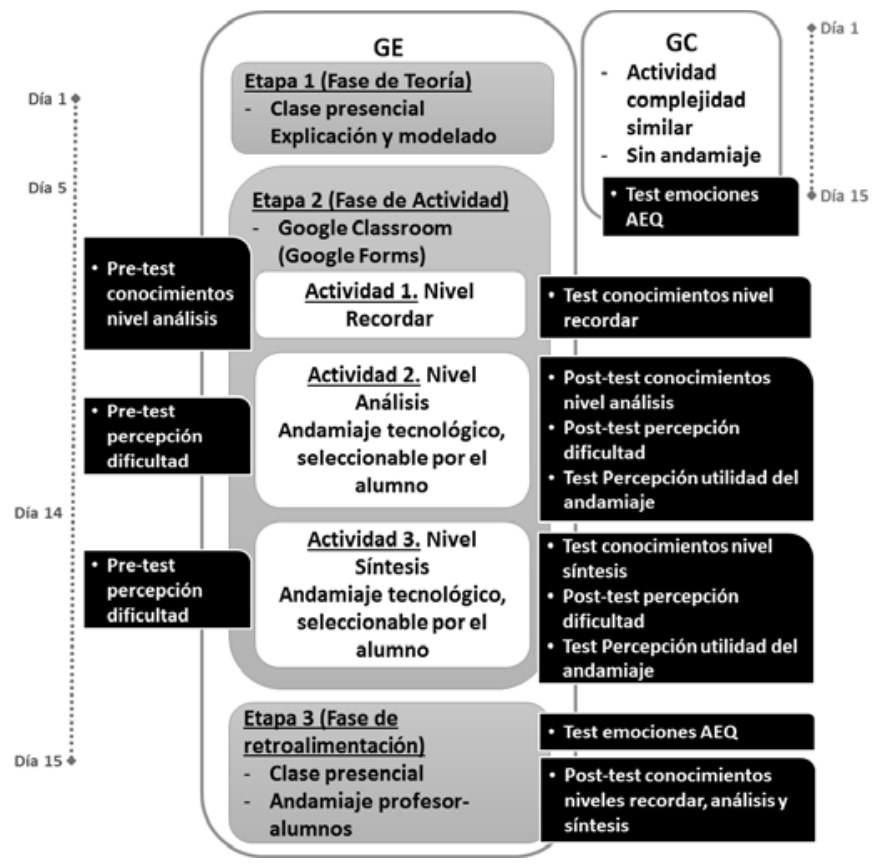

Figura 3 - Planificación de la intervención

\section{Resultados}

El análisis estadístico de los datos recogidos se realizó con ayuda del software IBM SPSS Statistics. Debido al tamaño muestral $(\mathrm{n}<30)$ y al rechazo de la hipótesis de normalidad (Prueba de Shapiro-Wilk) para la mayoría de las variables recogidas, se utilizaron test no paramétricos. A continuación, se exponen los resultados de manera detallada.

\section{A. Mejora en los resultados del aprendizaje}

Para el grupo experimental, se ha comparado en diferentes momentos el nivel de conocimiento alcanzado por los alumnos para distintos niveles de la Taxonomía de Bloom. La Tabla 1 muestra la estadística descriptiva de las variables relacionadas con el conocimiento.

Tabla 1 - Estadística descriptiva variables conocimiento

\begin{tabular}{|l|c|c|c|c|c|}
\hline \multicolumn{1}{|c|}{ Variable } & $\begin{array}{c}\text { Datos } \\
\text { válidos }\end{array}$ & Mín. & Máx. & Media & $\begin{array}{c}\text { Desv. } \\
\text { Típca }\end{array}$ \\
\hline ConocimientoRecordar_POS_INTER & 16 & 2.50 & 7.50 & 5.78 & 1.98 \\
\hline ConocimientoRecordar_POS_FINAL & 16 & 5.00 & 10.00 & 9.38 & 1.71 \\
\hline ConocimientoAnalisis_PRE & 16 & 3.00 & 10.00 & 6.44 & 3.01 \\
\hline ConocimientoAnalisis_POS_INTER & 12 & 2.33 & 10.00 & 6.39 & 3.33 \\
\hline ConocimientoAnalisis_POS_FINAL & 16 & 3.00 & 10.00 & 8.63 & 2.50 \\
\hline ConocimientoSintesis_POS_INTER & 11 & 1.40 & 7.00 & 4.04 & 2.11 \\
\hline ConocimientoSintesis_POS_FINAL & 15 & 6.00 & 10.00 & 8.93 & 1.16 \\
\hline
\end{tabular}

Se aplicaron cuatro pruebas de contraste de hipótesis de igualdad de distribuciones (Prueba de Wilcoxon) encontrando diferencias estadísticamente significativas $(\mathrm{p}<0.05)$ tal y como se muestra en la Tabla 2.

Tabla 2 - Contrastes sobre conocimiento 


\begin{tabular}{|c|c|c|}
\hline Contraste & Descripción & $\begin{array}{c}\text { p-valor (Prueba } \\
\text { de Wilcoxon) }\end{array}$ \\
\hline$\# 1$ & $\begin{array}{r}\text { ConocimientoRecordar_POS_INTER frente } \\
\text { ConocimientoRecordar_POS_FINAL }\end{array}$ & $\mathbf{0 . 0 0 1}$ \\
\hline$\# 2$ & $\begin{array}{r}\text { ConocimientoAnalisis_PRE frente } \\
\text { ConocimientoAnalisis_POS_INTER }\end{array}$ & 0.989 \\
\hline$\# 3$ & $\begin{array}{r}\text { ConocimientoAnalisis_PRE frente } \\
\text { ConocimientoAnalisis_POS_FINAL }\end{array}$ & $\mathbf{0 . 0 2 3}$ \\
\hline$\# 4$ & $\begin{array}{r}\text { ConocimientoSintesis_POS_INTER frente } \\
\text { ConocimientoSintesis_POS_FINAL }\end{array}$ & $\mathbf{0 . 0 0 5}$ \\
\hline
\end{tabular}

Se puede observar que el conocimiento final en cada uno de los tres niveles de Bloom (recordar, analizar y sintetizar) mejoraron tras la experiencia. Por tanto, podemos aceptar la hipótesis $\mathrm{H} 1 \mathrm{y}$ afirmar que el uso de andamiaje en el modelo de aprendizaje propuesto, mejora los resultados de aprendizaje en los niveles elevados de la taxonomía de Bloom.

\section{B. La percepción de dificultad y utilidad influye en las emociones y en los resultados de aprendizaje}

La Tabla 3 muestra las principales relaciones encontradas entre las variables recogidas del grupo experimental.

Tabla 3 - Principales relaciones lineales entre las variables

\begin{tabular}{|c|c|c|c|}
\hline Correlación & Variables relacionadas & $\begin{array}{l}\text { Rho de } \\
\text { Spearman }\end{array}$ & $\begin{array}{c}\mathrm{p}- \\
\text { valor }\end{array}$ \\
\hline C1 & Ansiedad y PercepDificultad_POS & +0.67 & 0.006 \\
\hline $\mathrm{C} 2$ & Ansiedad y PercepUtilidadScaff_TECNO & -0.56 & 0.061 \\
\hline C3 & $\begin{array}{c}\text { Desesperanza y } \\
\text { PercepUtilidadScaff_PROF }\end{array}$ & -0.58 & 0.019 \\
\hline $\mathrm{C} 4$ & Desesperanza y PercepDificultad_POS & +0.54 & 0.039 \\
\hline $\mathrm{C5}$ & $\begin{array}{l}\text { Enfado y post-test conocimientos final } \\
\text { (ConocimientoRecordar_POS_FINAL, } \\
\text { ConocimientoAnalisis_POS_FINAL, } \\
\text { ConocimientoSintesis_POS_FINAL) }\end{array}$ & -0.49 & 0.054 \\
\hline C6 & Disfrute y PercepUtilidadScaff_TECNO & +0.61 & 0.035 \\
\hline C7 & $\begin{array}{c}\text { PercepDificultad_PRE y } \\
\text { ConocimientoSintesis_POS_INTER }\end{array}$ & -0.57 & 0.066 \\
\hline $\mathrm{C} 8$ & $\begin{array}{l}\text { ConocimientoSintesis_POS_INTER y } \\
\text { PercepUtilidadScaff_PROF }\end{array}$ & -0.46 & 0.159 \\
\hline
\end{tabular}

Se puede observar que las emociones negativas influyen en general en las percepciones y en el conocimiento, en concreto:

- $\quad$ La ansiedad influye en la percepción de dificultad de la tarea y de la utilidad del andamiaje tecnológico (Tabla 3, C1 y C2).

- La desesperanza influye en la percepción de la dificultad y utilidad del andamiaje del profesor (Tabla 3, C3 y C4).

- El enfado influye en la adquisición de conocimiento (Tabla 3, C5).

Además, las emociones positivas también influyen: el disfrute repercute en la percepción de utilidad del andamiaje tecnológico (Tabla 3, C6). Por último, la percepción de dificultad $y$ de utilidad influye en la adquisición del conocimiento (Tabla 3, C7 y C8). En consecuencia, los resultados afirman que la percepción de dificultad de la tarea y de la utilidad del andamiaje, aspectos de facilidad de uso (usability) del modelo propuesto, están relacionadas con las emociones, por lo que se puede aceptar la hipótesis H2. Además, como hallazgo adicional y teniendo en cuenta las correlaciones entre las variables de percepción y de resultados de aprendizaje, podemos afirmar que las percepciones de dificultad y de utilidad (aspectos de la facilidad de uso), también influyen en los resultados de aprendizaje.

\section{Mejora del estado emocional}

Se han comparado las emociones sentidas por el grupo experimental y por el grupo control después de realizar la experiencia. En la Figura $\mathbf{4}$ Error! No se encuentra el origen de la referencia. se muestra la media por grupos de las variables de las emociones, donde se puede ver que el grupo experimental presenta menores valores en las emociones negativas y mayor en las positivas.

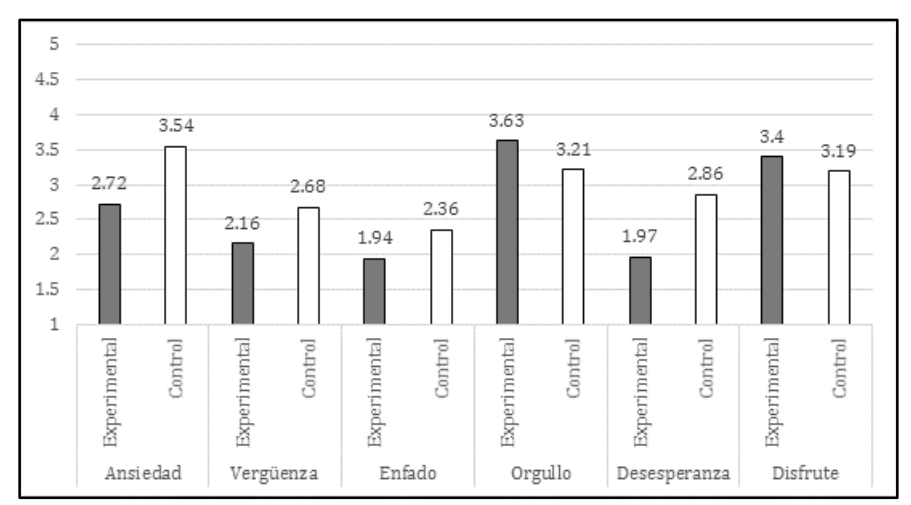

Figura 4-Media de las variables sobre emociones

En la Tabla 4 se muestra el contraste de igualdad entre grupos (Prueba de U-Mann Whitney) encontrando diferencias estadísticamente significativas $(p<0.05)$. Se puede observar que los niveles de ansiedad y desesperanza son significativamente menores en el grupo experimental que en el grupo de control (marcados en negrita en la Tabla 4).

Tabla 4 - Contrastes sobre emociones entre grupos

\begin{tabular}{|c|c|c|}
\hline Contraste & Descripción & $\begin{array}{c}\text { p-valor (Prueba } \\
\text { U-Mann Whitney) }\end{array}$ \\
\hline$\# 7$ & Ansiedad GE frente GC & $\mathbf{0 . 0 2 5}$ \\
\hline$\# 8$ & Vergüenza GE frente GC & 0.294 \\
\hline$\# 9$ & Enfado GE frente GC & 0.240 \\
\hline$\# 10$ & Orgullo GE frente GC & 0.334 \\
\hline$\# 11$ & Desesperanza GE frente GC & $\mathbf{0 . 0 1 2}$ \\
\hline$\# 12$ & Disfrute GE frente GC & 0.552 \\
\hline
\end{tabular}

También se han comparado las percepciones de los alumnos sobre la dificultad de las actividades (antes y después de realizarlas con andamiaje) y la percepción de utilidad del andamiaje tecnológico frente al andamiaje profesor-alumnos. En la Tabla 5 se muestra la estadística descriptiva de estas variables de percepción.

Tabla 5 - Estadística descriptiva de las variables de percepción del estudiante 


\begin{tabular}{|c|c|c|c|c|c|}
\hline Variable & $\begin{array}{c}\text { Datos } \\
\text { válidos }\end{array}$ & Mín. & Máx. & Media & $\begin{array}{c}\text { Desv. } \\
\text { Típca }\end{array}$ \\
\hline PercepDificultad_PRE & 12 & 3.00 & 4.75 & 4.06 & 0.54 \\
\hline PercepDificultad_POS & 12 & 1.00 & 4.00 & 1.83 & 0.94 \\
\hline PercepUtilidadScaff_TECNO & 12 & 1.50 & 4.50 & 2.96 & 1.21 \\
\hline PercepUtilidadScaff_PROF & 12 & 3.50 & 5.00 & 4.29 & 0.45 \\
\hline
\end{tabular}

En la Tabla 6 se muestran los resultados de los dos contrastes de hipótesis sobre las percepciones (Prueba de Wilcoxon), encontrando diferencias estadísticamente significativas $(\mathrm{p}<$ $0.01)$.

Tabla 6 - Contrastes sobre percepciones

\begin{tabular}{|c|c|c|}
\hline Contraste & Descripción & $\begin{array}{c}\mathbf{p} \text {-valor (Prueba } \\
\text { de Wilcoxon) }\end{array}$ \\
\hline$\# 5$ & $\begin{array}{r}\text { PercepDificultad_PRE frente } \\
\text { PercepDificultad_POS }\end{array}$ & 0.002 \\
\hline$\# 6$ & $\begin{array}{c}\text { PercepUtilidadScaff_TECNO frente } \\
\text { PercepUtilidadScaff_PROF }\end{array}$ & 0.007 \\
\hline
\end{tabular}

En consecuencia, la percepción de dificultad después de realizar la experiencia completa con andamiaje disminuye. Además, los alumnos perciben mayor utilidad en el andamiaje profesor-alumnos que en el andamiaje tecnológico. Por tanto, se acepta la hipótesis H3 afirmando que el modelo de aprendizaje propuesto mejora el estado emocional y reduce la percepción de dificultad del estudiante ante la tarea a realizar, aspectos importantes de la facilidad de uso (usability).

\section{CONCLUSIONES}

En este artículo se presenta un modelo de aprendizaje basado en andamiaje y su aplicación en el contexto pandémico de la COVID-19. Para validar la efectividad de aprendizaje y la facilidad de uso del modelo propuesto se ha desarrollado un soporte digital basado en plataformas abiertas y se ha realizado una experiencia con alumnos de Formación Profesional de Grado Superior. Son dos los hallazgos principales encontrados como resultado del estudio realizado. En primer lugar, se ha hallado que el uso de andamiaje facilita la adquisición de conocimiento en los niveles superiores de complejidad de la taxonomía de Bloom, mejora el estado emocional del alumno y reduce la percepción de dificultad que tiene de la tarea. En segundo lugar, se ha encontrado que la percepción de dificultad que tiene el estudiante de la tarea y la percepción de utilidad sobre el andamiaje empleado están relacionados con las emociones que experimenta en el desarrollo del aprendizaje.

Como trabajo futuro, sería necesario replicar la experiencia con un mayor tamaño muestral que permita consolidar las conclusiones obtenidas en el actual estudio y analizar el comportamiento de las emociones a lo largo del aprendizaje, no solo al finalizar la tarea, sino antes y durante la misma.

\section{AGRADECIMIENTOS}

Se agradece el apoyo de los estudiantes que participaron y de los docentes del IES Julio Verne de Leganés que permitieron la realización de este estudio. Este trabajo ha sido financiado gracias al proyecto e-Madrid (Ref. P2018/TCS-4307) con fondos FSE y FEDER.

\section{REFERENCIAS}

Belland, B. R. (2017). Instructional Scaffolding in STEM Education. Springer International Publishing. https://doi.org/10.1007/978-3-319-02565-0

Bloom, B. S. (1956). Handbook I, cognitive domain. Taxonomy of educational objectives: the classification of educational goals. New York: Longman.

Delors, Jacques (1994). Los cuatro pilares de la educación, en La Educación encierra un tesoro. México: El Correo de la UNESCO, pp. 91-103

Kim, N. J., Belland, B. R., \& Walker, A. E. (2018). Effectiveness of Computer-Based Scaffolding in the Context of Problem-Based Learning for Stem Education: Bayesian Meta-analysis. Educational Psychology Review, 30(2), 397-429. https://doi.org/10.1007/s10648017-9419-1

Krathwohl, D. R. (2002). A Revision of Bloom's Taxonomy: An Overview. Theory Into Practice, 41(4), 212-218. https://doi.org/10.1207/s15430421tip4104_2

Lacave, C., Velázquez-Iturbide, J. A., Paredes-Velasco, M., \& Molina, A. I. (2020). Analyzing the influence of a visualization system on students' emotions: An empirical case study. Computers \& Education, 149, 103817. http://dx.doi.org/10.1016/j.compedu.2020.103817 7

Paoloni, P., Vaja, A., \& Muñoz, V. (2014). Reliability and validity of the Achievement Emotions Questionnaire. A study of Argentinean university students. Electronic Journal of Research in Educational Psychology, 12, 671692. https://doi.org/10.14204/ejrep.34.14088

Pérez, B. C., \& Aleixandre, M. P. J. (2018). Influencia de distintas estrategias de andamiaje para promover la participación del alumnado de secundaria en las prácticas científicas. Enseñanza de las Ciencias. Revista de investigación y experiencias didácticas, 36(2), 23-42. https://doi.org/10.5565/rev/ensciencias. 2241

Real Decreto 1147/2011, de 29 de julio, por el que se establece la ordenación general de la formación profesional del sistema educativo. Boletín Oficial del Estado 201113118. Recuperado 16 de mayo de 2021, de https://www.boe.es/buscar/doc.php?id=BOE-A-201113118

Van de Pol, J., Volman, M., \& Beishuizen, J. (2010). Scaffolding in Teacher-Student Interaction: A Decade of Research. Educational Psychology Review, 22(3), 271296. https://doi.org/10.1007/s10648-010-9127-6

Vigotsky, L. S. (1979). El Desarrollo de los Procesos Psicológicos Superiores. Barcelona: Crítica, D. L.

Wood, D., Bruner, J. S., \& Ross, G. (1976). The Role of Tutoring in Problem Solving. Journal of Child Psychology and Psychiatry, 17(2), 89-100. https://doi.org/10.1111/j.1469-7610.1976.tb00381.x 